\title{
Componentes predominantes de la acción tutorial en estudiantes universitarios
}

\section{Predominant Components of the Tutorial Action in University Students}

\author{
Julia Guerrero-Ramírez \\ Universidad César Vallejo, Lima, Perú \\ ORCID: https://orcid.org/0000-0002-2507-5063 \\ Doris Fuster-Guillén* \\ Universidad Nacional Mayor de San Marcos, Lima, Perú \\ ORCID: https://orcid.org/0000-0002-7889-2243 \\ Eric Gálvez-Suarez \\ Universidad Privada del Norte, Lima, Perú \\ ORCID: https://orcid.org/0000-0002-7642-5019 \\ Yolvi Ocaña-Fernández \\ Universidad Nacional Mayor de San Marcos, Lima, Perú \\ ORCID: https://orcid.org/0000-0002-2566-6875

\section{Dante Aguinaga-Villegas} \\ Universidad Nacional Mayor de San Marcos, Lima, Perú \\ ORCID: https://orcid.org/0000-0001-5166-0509
}

Recibido 10-08-18 Revisado 30-10-18 Aprobado 11-03-19 En línea 12-03-19

*Correspondencia

Email: dorisfusterguillen@gmail.com

\author{
Citar como:
Guerrero-Ramírez, J., Fuster-Guillén, D., Gálvez-Suarez, E., Ocaña-Fernández, Y., \& Aguinaga-Villegas, D. (2019). Componentes predominantes de la acción tutorial en estudiantes universitarios. Propósitos y Representaciones, 7(2), 304-324. doi:
http://dx.doi.org/10.20511/pyr2019.v7n2.300




\section{Resumen}

La investigación relacionada a la práctica de la acción tutorial en estudiantes universitarios, tuvo como objetivo determinar el componente predominante de la tutoría, reconociendo que la tutoría en la universidad está cobrando valor y significancia en beneficio de los discentes, como área de apoyo, orientación y cobertura durante el proceder educativo en sus distintas dimensiones académicas, profesional y personal. El estudio corresponde al enfoque cuantitativo, de método hipotético deductivo tipo sustantivo, de diseño no experimental, de corte transversal o transaccional y explicativa, con una población de 2629 estudiantes cuya muestra representativa fue de 335 participantes. Los resultados del mismo, demostraron que el 67\% de los estudiantes consideraron que la acción tutorial que recibieron fue adecuada, mientras que el 33\%, asimismo establece que la dimensión predominante destacada fue el desarrollo académico, se concluye que la acción tutorial sí tuvo presencia significativa y predominante al haber sido considerada como adecuada dentro del desarrollo de la vida universitaria en estudiantes en formación; situación que confirmó y revaloró por la atención a distintas necesidades e intereses por parte del alumnado respondiendo a una educación de desarrollo integral.

Palabras clave: Acción tutorial; Dimensión personal; Dimensión académica; Dimensión profesional.

\section{Summary}

The research related to the practice of tutorial action in university students, aimed to determine the predominant component of tutorial, recognizing that tutorial in the university is gaining value and significance for the benefit of the students, as a support area, orientation and coverage during the educational procedure in its different academic, professional and personal dimensions. The study corresponds to the quantitative approach, deductive hypothetical and substantive method, non-experimental, cross-sectional or transactional and explanatory design, with a population of 2629 students whose representative sample was 335 participants. The results of the same showed that $67 \%$ of the students considered that the tutorial action they received was adequate, while $33 \%$, also states that the predominant dimension was the academic development. It is concluded that the tutorial action did have a significant and predominant presence since it was considered as adequate within the development of university life in undergraduate students; situation that confirmed and revalued the attention to different needs and interests by the students responding to an education of integral development.

Keywords: Tutorial Action; Personal Dimension; Academic Dimension; Professional Dimension.

\section{Introducción}

La adopción de la tutoría en el marco universitario ha ido cobrando valor y relevancia en los propios protagonistas, en los docentes como estudiantes, así como en los responsables de la misma. El contexto del Espacio Europeo de Educación Superior (EEES) señaló que la función educadora debería mantenerse con una visión amplia que involucre el trabajo tutorial, pero que en la realidad solo se asume como un ideal y secundaria y que no se le involucre en el proceso de aprendizaje del estudiante (Álvarez, 2013, p. 101). La percepción de la misma acción tutorial es variada; en México, Caldera, Carranza, Jiménez y Pérez (2015) en un estudio en la universidad de Guadalajara reconocieron las actitudes que los estudiantes universitarios reflejan ante la tutoría, resultados que revelaron actitudes positivas; su desarrollo práctico tiene ya un tiempo prudencial de ejecución, los alumnos en estos programas educativos, tienen claridad y conocimiento de las razones por los cuales la tutoría se viene realizando. En el caso peruano, la entidad del Programa Nacional de Becas y Crédito Educativo - PRONABEC (2012, p. 23-26) muestran resultados que preocupan y los que reflejan el poco interés por asuntos relacionados a la acción tutorial en el nivel superior, se muestran otros trabajos anteriores referidos a la acción 
tutorial o tutoría a nivel superior como, Lemus, Torres, Serrano y Gúzman (2015), reconocieron problemáticas de mayor incidencia en relación a juicios de evaluación aplicados para certificar el aprendizaje, se sugirió implantar un modelo de apoyo ocurrente a la acción tutorial, incidiendo en la productividad académica, a la estima personal y en trabajo con los demás asimismo Amor (2012 ratificó que la tutoría no estuvo siendo abordada en su total importancia, solo fue considerada como un elemento adicional y que no se relaciona con el proceso de aprendizaje de los estudiante, todas sus conclusiones abordaron una preocupación y problemática real acerca de los planes de tutoría y orientación en el nivel universitario, ya que no solo basta con conocer o desear mejorar, sino de establecer programas claros para la participación activa por parte de docentes tutores y estudiantes. También Álvarez (2013, ante los cambios que contrajo el modelo del espacio europeo de educación superior en relación a la labor de la tutoría, situándolo en una posición relevante en los sistemas de garantía interna de calidad, parte de su estudio se enfocó en el rol que desempeña el docente en cuanto a su función tutorial con el alumnado y a la colaboración e implicación del mismo en el acrecentamiento de un sistema de tutoría de carrera en las titulaciones, concluyendo también que la función tutorial se dedujo solo como una labor adicional o secundaria, no necesariamente involucrada al proceso de aprendizaje del tutorado, también que los distintos agentes involucrados deberían componer un buen funcionamiento de sistemas de orientación y programas de tutoría. En el caso de Gil, Martínez, Tunnicliffe y Moneo (2013), identificó que con el establecimiento del EEES, las instituciones superiores consideraron en su realidad dentro de su proceso de enseñanza aprendizaje propósitos u objetivos de acción tutorial y la estimación de los efectos del mismo pondría en evidente el menester de mejorar de forma constante para el logro de una mayor efectividad, indicios de evaluación y estudio que motivó a la investigación, dando a conocer que los estudiantes sí respaldan una cobertura tutorial, la aceptan y la valoran, en este caso fue que otorgan mayor influencia para el desarrollo profesional, que en lo personal o académico, y más aún si se podría considerar mantener el mismo lazo de comunicación con un mismo tutor; calificación que apremió una importante intervención en el desarrollo personal, académico y profesional, el cual también se consideró e la investigación, pero en otra realidad atendiendo a necesidades e intereses distintos. Por su lado Romo y Romero (2015) defendieron que la tutoría no alcanza todas las responsabilidades en fomentar un cambio radical a situaciones de riesgo en un discente o de que conserve un historial intachable; pero sí se asegura que la ejecución eficiente de un programa de tutoría ordena mantener una constante evaluación con fines de mejora y sobre todo con la cobertura de las autoridades y de más servicios presentados por instituciones de nivel superior, las mismas situaciones vistas en la experiencia docente y la labor tutorial, que crearon inquietudes y la necesidad de saber las verdaderas exigencia que demanda la acción tutorial en los distintos componentes académico, personal y profesional; por ello el trabajo establece el problema ¿Cuál es la dimensión predominante en la acción tutorial en estudiantes universitarios?.

\section{Orientación educativa y tutoría.}

Respecto a ello Pantoja y Campoy (2009), refieren que en muchas ocasiones la orientación educativa y la tutoría son relacionadas, el autor reconoció que la orientación educativa es un componente adjunto a la misma educación integrando así la sugerencia curricular, interpretada y conducida a todos los lineamientos del aprendizaje y avance, asistiendo al establecimiento y realización de una educación global al contribuir con el asesoramiento y soporte técnico en funciones personalizadas para la educación, que muestran un enfoque atento a la variedad de habilidades y motivaciones que puedan presentar los discentes. La orientación educativa y acción tutorial se encuentran estrechamente ligadas, de tal manera que conformar parte de la función docente y por tal del mismo tutor. (p. 271). Los autores reconocen una relación cercana en ambas, aunque a la orientación se le atribuya un plano más general y preparado para instancias particulares y que puede asumir roles en distintos aspectos de la persona ante sus futuras decisiones, la tutoría, será vista como una parte del procedimiento de orientación en el proceso educativo pero que también con funciones en particular. 


\section{Acción tutorial.}

Gil, Martínez, Tunnicliffe y Moneo (2013), refieren que la acción tutorial es una función necesaria presente en todos los niveles educativos, en procesos de desarrollo personal, académico y profesional... que se materializa mediante la implementación de planes de acción tutorial, que deben ser flexibles y adaptables a las situaciones y recursos disponibles. (p.65), es por ello, que se tomó como referencia para la delimitación de las dimensiones en acción tutorial, lo personal, académico y profesional componentes claves en la tutoría universitaria, al respecto Álvarez (2014) nombrado por Lobato y Guerra (2016) mencionó que la "tutoría universitaria es una función de acompañamiento, de orientación y de apoyo al alumnado en su proceso de personalización de los aprendizajes y del desarrollo de las competencias, tanto a nivel personal como profesional, a lo largo de su trayectoria, con el horizonte dinámico del proyecto de vida, un proyecto personal y profesional”. (p. 383). De la misma forma, Pantoja y Campoy (2009) para el ámbito de la Universidad anunciaron que:

La tutoría debe discernirse pieza activa dentro de los subsistemas de los sistemas o áreas de la universidad de apoyo al estudiante en el campo de su aprendizaje. La tutoría universitaria debe asumir cambios como una nueva forma de planificar el tiempo, plantearse metas más rigurosas, estar integrada al proceso de enseñanzaaprendizaje, por tal, es un suplemento la labor de clase, que se lleva a cabo en espacios diversos y está desempeñada por diferentes agentes. (p.272).

Para Pantoja y Campoy (2009) la tutoría permite un vínculo cercano, es decir una relación personalizada con el estudiante, cumpliendo de esa manera la función formadora y de acompañamiento que permita al estudiante conocerse así mismo, sabrá la forma de cómo actúa reflexionando en ello o piensa en relación a distintas situaciones; valore sus cualidades y capacidades; que adquiera una visión de la sociedad en la que vive; que aprenda a vivir en sociedad; que integre e implique al estudiante en la vida académica; que pueda lograr un adecuado manejo de sus emociones; aprender a construir su proyecto académico, profesional y personal. Para esto PRONABEC en el 2012, mencionaron que la educación a nivel universitaria y tecnológica, poseen como objeto la atención a jóvenes y adultos con peculiaridades precisas derivadas de su experiencia en distintas áreas, razón por el que el sistema de tutoría se sustenta en bases androgógicas que fortalecen y vigorizan a la propuesta, estos fundamentos relacionados con la tutoría son la madurez emocional y autorregulación, el pensamiento crítico, la autodeterminación que desarrolla su autonomía, el pensamiento innovador y sobre todo el compromiso social, todo esto muestra el enfoque de acción de la tutoría. La misma que Caldera, Carranza, Jiménez, y Pérez (2015) desarrollaron la tutoría en dos modalidades; una es la modalidad o tutoría individual, centrada en la atención personalizada en beneficio y cobertura al estudiante, esta relación puede ser considerada de modo presencial o virtual, acrecentando vínculos de mayor seguridad y confianza de manera que pueda consolidar su autoestima; la otra modalidad es la tutoría grupal, donde el docente tutor puede interactuar con el grupo de estudiantes de modo presencial o a distancia, requiere también de organización y planificación para establecer fechas u horarios de congregación (presencial o virtual), y temáticas que puedan motivar al grupo para participar de manera activa, esta modalidad tiene el atributo de acceder a mayor número de tutorados a menor costo de tiempos y espacios.

\section{Componentes de la acción tutorial.}

Esta será revelada en las siguientes funciones de la acción tutorial planteada por Quintanal y Miraflores (2013), la acción tutorial deberán ser establecidas a partir de lineamientos que permitan con mayor claridad, definidas en cuatro aspectos relevantes, primero adoptada como guía de incorporación al medio universitario (primeros asignaturas), donde el tutor será la persona más cercana a los estudiantes en esta adaptación para alcanzar sus dudas desde sus inicios al ambiente universitario; segundo el apoyo académico (enseñar a estudiar, motivar, informar sobre la 
asignatura en cuestión, etc.) evitando la deserción o abandono de estudios por problemas académicos o vocacionales, el tutor no es el docente de la asignatura, pero es quien pueda brindar orientación en base a las asignaturas designadas. Tercero la orientación y atención a dificultades personales (formar la autonomía y responsabilidad, dar a conocer sobre los derechos y obligaciones, encauzar sus demandas e inquietudes, instruir en la forma de resolver conflictos o impedimentos, et.). mediante el diálogo y estrecha relación con el tutor designado y como último la orientación profesional (probables salidas profesionales, informar de cursos, seminarios, congresos, bibliografías actualizadas, etc), manteniendo una visión más general y de la importante labor y función que puede establecer esta acción tutorial y el impacto que puede tener si realmente cada tutor está capacitado y es el idóneo para el trabajo tutorial; por tal la importancia de la tutoría en la universidad como refiere Muñoz y Gairín (2013) que la orientación y cobertura al alumno en el transcurso de su estadía universitaria es asunto de especial y actual tendencia para los encargados institucionales, tanto preocupados por la capción, contención y progreso del provecho académico. Al pasar del tiempo el mismo estudiante se convertirá en el agente activo en la vida universitaria, desde el participar en actividades extracurriculares, reflexionar en su estilo o forma de aprendizaje, asignaturas, ser orientado en la mejora de su aprendizaje, entre otros, mostrando un riqueza un desarrollo personal, académico y profesional, es la tutoría quien pueda contribuir de forma más cercana a distintas acciones donde los estudiantes tengan la mayor cobertura, no permitiendo la separación o abandono de sus estudios. Fue necesario conocer y estudiar aquellos ámbitos de acción tutorial, en este caso Gil, Martínez, Tunnicliffe y Moneo (2013), en su estudio compartieron sus experiencias de trabajo en relación a la acción tutorial y el impacto que ha causado por 6 años en la Universidad de San Jorge tras la implementación de un plan de acción, proponiendo conocer y evaluar las necesidades específicas en los estudiantes necesitan cubrir en tres ámbito de desarrollo para el alcance de una maduración integral; se menciona la orientación a nivel personal, aportando al desarrollo de su personalidad - identidad, también el orientar en aspectos académicos, acrecentando habilidades inherentes de aprendizaje autónomo y por último la orientación profesional al estudiante, mostrando el acompañamiento del aprendizaje en el ambiente laboral en relación a la inclusión laboral y autoempleo; esto con el fin en que cada uno pueda visionar y planificar su proyecto de vida. Todo este impacto de labor en distintos ámbitos, también exigirá un nuevo rol del profesorado universitario para el cumplimento de su labor, tanto como docente de asignatura y como en su labor tutorial. La intervención del docente tutor, con el desarrollo de competencias que puedan mejorar su actuación; Quintanal y Miraflores (2013) resaltaron las propuestas de Zabalza, en relación a competencias que todo docente deberá desarrollar para la mejora de su actuación docente y tutorial; entre las que menciona el facilitar una buena adaptación al contexto de la enseñanza universitaria con la intención de prever realidades de incertidumbre, abandono, frustración, permuta o prolongación de los estudios; segundo aconsejar al alumnado en relación a lo que puede hacer y cómo actuar en los distintos momentos de su proceso formativo universitario; también el poder orientar el aprendizaje del aprendiz mediante el desarrollo de distintos programas específicos en recomendación de actividades según las necesidades de cada estudiante; desarrollar estrategias de labor intelectual y de aprendizaje autónomo desde las asignaturas particulares, explicar las consecuencias de los contenidos curriculares para el buen desempeño profesional, informar sobre asuntos académicos, administrativos y profesionales que estén al alcance del tutor, facilitar tomas de decisiones apropiadas en aspectos académicos y profesionales y las consecuencias que podrían mostrarse; todo ello encierra un punto de mayor incapie a mayores estudios a detalle.

\section{Método}

El diseño de método de investigación radica en un enfoque cuantitativo, al seguir una secuencia ordenada, desde el identificar una problemática hasta obtener resultados y conclusiones determinantes de la investigación; de método hipotético deductivo, que connota desde una visión general en la investigación para obtener puntos específicos en la misma; el tipo de estudio acorde fue Sustantivo, en que Sánchez y Reyes (2015) hicieron referencia que se orienta a describir, explicar, predecir o retrodecir la realidad, con lo cual se va en búsqueda de principios y leyes generales que permita organizar una teoría científica. De diseño No experimental, pues no hubo 
manipulación de la variable acción tutorial, sino que se le consideró en su naturalidad, en el entorno de estudio con estudiantes universitarios, de forma que permitió conocer el impacto de la misma de la variable en el proceder del estudio, a su vez, de corte transversal o transaccional, pues los datos necesarios fueron derivados en un solo momento, es decir una solo intervención en un tiempo definido con la muestra. Al ser de tipo sustantiva y no experimental, también será de tipo explicativa, Sánchez y Reyes (2015) la definieron como aquella que está orientada al descubrimiento de los factores causales que han podido incidir o afectar la ocurrencia del fenómeno. Responde a las preguntas ¿Por qué se presenta así el fenómeno X?, ¿Cuáles son los factores o variables que están afectando X? (p. 39). De esta forma la misma intervención de tipo explicativa permitió saber y conocer el desenvolvimiento de la acción tutorial, diagnosticando para que en las recomendaciones se pueda proponer nuevas alternativas detalladas, conociendo el indicador o dimensión predominante, frente al estudio y al resultado de la influencia de distintos factores que se interponen el contexto.

La población fueron estudiantes de la universidad privada César Vallejo que tiene insertado el programa de tutoría. El total de la población durante el periodo académico 2017-I fueron de 2629 estudiantes en los turnos mañana, tarde y noche; donde todos los involucrados tuvieron las mismas posibilidades de pertenecer a la muestra. Para la muestra representativa mediante el muestreo probabilístico se aplicó la fórmula estadística de Arkin y Colton, siendo esta de 335 estudiantes que participaron en el estudio a través del muestreo aleatorio simple. Se utilizó la técnica de la encuesta que resultó la más pertinente para el estudio en relación a la acción tutoría, el instrumento a disposición fue la escala, donde cada estudiante pudo emitir un valor de grado de importancia de 1 al 10 en un total de 26 ítems, subdivididos y organizados en tres dimensiones (personal, académico y profesional). El instrumento fue remitido en la validez de contenido fue realizado mediante juicio de expertos y la validez de constructo realizado a través del análisis factorial. Para la confiabilidad del instrumento se utilizó el Alpha de Crombach, ya que los índices y rangos correspondieron a una escala ordinal. Para la normalidad de los datos se utilizó Kolmogiow Smirnov porque la muestra fue conformada por más de 50 sujetos.

\section{Resultados}

En los resultados descriptivos, se evidenció que el $67 \%$ de los estudiantes de la universidad César Vallejo - Lima Norte consideraron que la acción tutorial recibida fue adecuada, mientras que el $33 \%$ de los mismos consideraron que la acción tutorial que reciben fue inadecuada y regular

Tabla 1.

La acción tutorial en estudiantes de la universidad César Vallejo- Lima Norte

\begin{tabular}{lcc}
\hline Nivel & Frecuencia & Porcentaje \\
\hline Inadecuada & 27 & $8 \%$ \\
Regular & 85 & $25 \%$ \\
Adecuada & 223 & $67 \%$ \\
Total & 335 & $100 \%$ \\
\hline
\end{tabular}

En relación con la contrastación de las hipótesis que se realizó mediante la regresión logística, puesto que la prueba de normalidad indicó que no presentó normalidad, ya que el valor de los datos fue menor al valor de significación teórica, es por ello que se escoge una prueba no paramétrica. 
Tabla 2.

Coeficientes de la regresión logística de las dimensiones de la acción tutorial en estudiantes de la universidad César Vallejo- Lima Norte.

\begin{tabular}{lcccccc}
\hline \multirow{1}{*}{ Indicador } & $\mathrm{B}$ & Error estándar & Sig. & $\operatorname{Exp}(\mathrm{B})$ & \multicolumn{2}{c}{ 95\% C.I. para EXP(B) } \\
\cline { 6 - 7 } & & & & & Inferior & Superior \\
\hline $\begin{array}{l}\text { Desarrollo personal } \\
\begin{array}{l}\text { Desarrollo } \\
\text { académico }\end{array}\end{array}$ & -.048 & 0.131 & 0.715 & 0.953 & 0.737 & 1.233 \\
$\begin{array}{l}\text { Desarrollo } \\
\text { profesional }\end{array}$ & -.082 & 0.144 & 0.569 & 0.922 & 0.696 & 1.221 \\
\hline
\end{tabular}

La dimensión de mayor peso es el desarrollo académico, pero que ésta a su vez no es significativa estadísticamente.

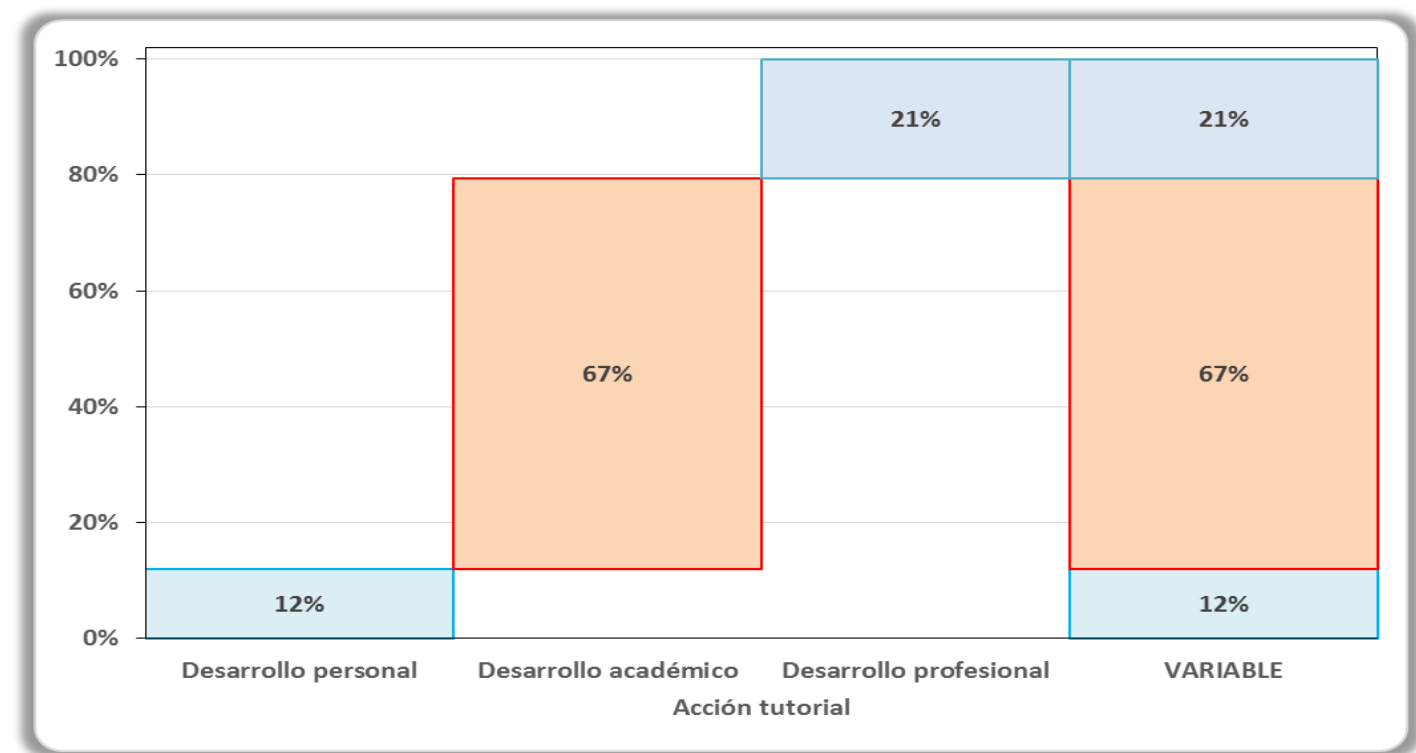

Figura 1. Pesos de las dimensiones de la acción tutorial en estudiantes de la Universidad César Vallejo- Lima Norte.

Se evidenció que la dimensión que registra mayor predominancia en la acción tutorial fue el desarrollo académico, Sin embargo, la dimensión predominante de desarrollo académico es considerado un factor de riesgo ya que su valor de odds ratio $\langle\operatorname{Exp}(B)>$ es mayor a 1 , convirtiéndose así en la dimensión que necesita mayor atención, ya que, si no se vela por ella, la acción tutorial tendría mayores dificultades que los previstos. 
Tabla 3.

Coeficientes de la regresión logística de los indicadores de la dimensión desarrollo académico en estudiantes de la universidad César Vallejo- Lima Norte.

\begin{tabular}{|c|c|c|c|c|c|c|}
\hline \multirow{2}{*}{$\begin{array}{c}\text { Indicadores del } \\
\text { desarrollo académico }\end{array}$} & \multirow[t]{2}{*}{ B } & \multirow{2}{*}{$\begin{array}{c}\text { Error } \\
\text { estándar }\end{array}$} & \multirow[t]{2}{*}{ Sig. } & \multirow[t]{2}{*}{$\operatorname{Exp}(B)$} & \multicolumn{2}{|c|}{$\begin{array}{c}\text { 95\% C.I. para } \\
\text { EXP(B) }\end{array}$} \\
\hline & & & & & Inferior & Superior \\
\hline $\begin{array}{l}\text { Adaptarme al } \\
\text { funcionamiento de la } \\
\text { universidad. }\end{array}$ & 0.270 & 0.112 & 0.016 & 1.310 & 1.052 & 1.630 \\
\hline $\begin{array}{l}\text { Aprender a gestionar mi } \\
\text { tiempo para el estudio y } \\
\text { realización de trabajos. } \\
\text { Desarrollar estrategias de }\end{array}$ & .009 & 0.142 & 0.947 & 1.009 & 0.765 & 1.332 \\
\hline $\begin{array}{l}\text { Desarrollar estrategias de } \\
\text { aprendizaje autónomo } \\
\text { para tener éxito } \\
\text { académico. }\end{array}$ & -0.225 & 0.147 & 0.126 & 0.798 & 0.598 & 1.065 \\
\hline $\begin{array}{l}\text { Aprender a investigar y } \\
\text { realizar trabajos } \\
\text { académicos. }\end{array}$ & 0.345 & 0.117 & .003 & 1.412 & 1.123 & 1.776 \\
\hline $\begin{array}{l}\text { Tomar conciencia de mi } \\
\text { capacidad de aprendizaje } \\
\text { para mejorar mi } \\
\text { rendimiento. }\end{array}$ & -0.281 & 0.116 & .016 & 0.755 & 0.601 & 0.948 \\
\hline
\end{tabular}

El indicador de mayor peso fue aprender a investigar y realizar trabajos académicos y el segundo en predominancia es el indicador tomar conciencia de mi capacidad de aprendizaje para mejorar mi rendimiento es signifcativa.

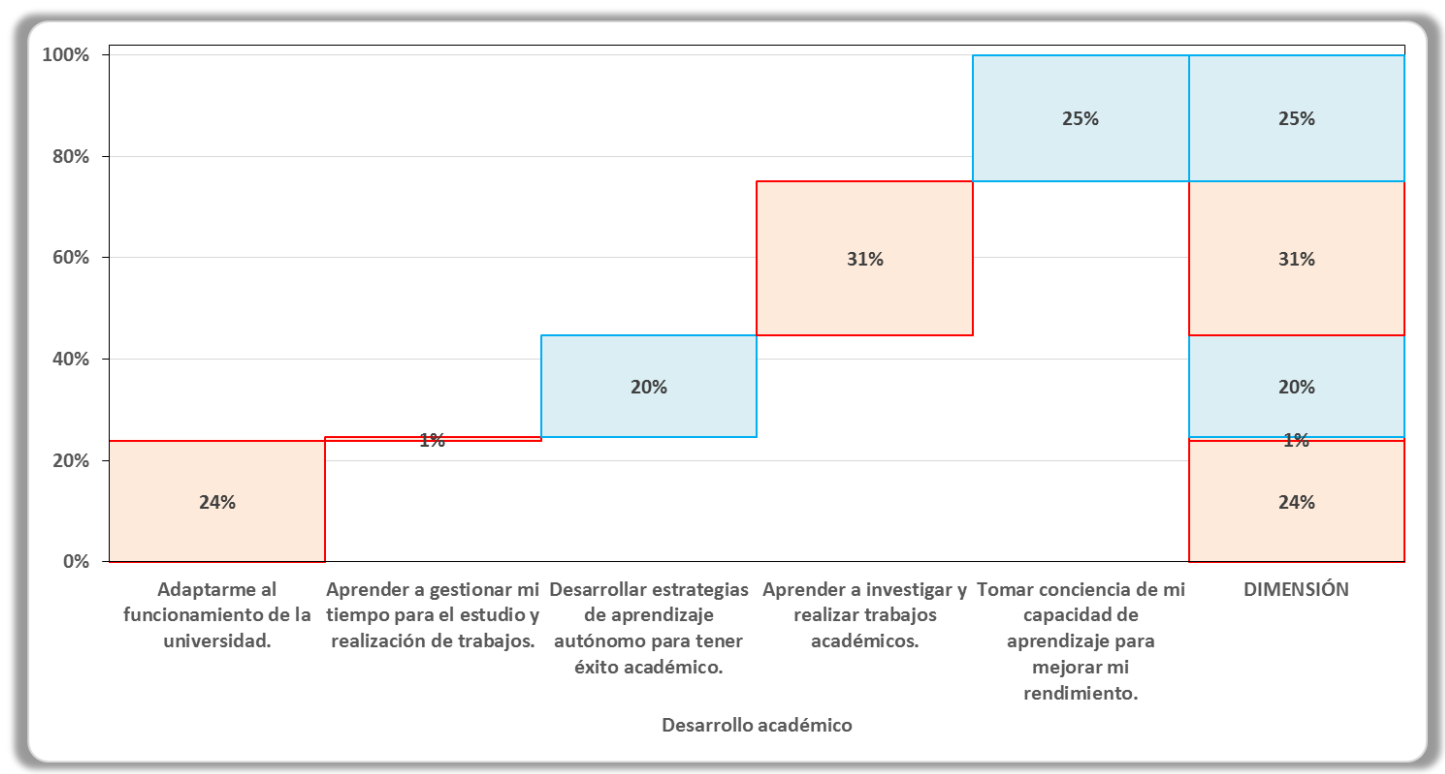

Figura 3. Pesos de los indicadores de la dimensión desarrollo académico en estudiantes de la universidad César Vallejo- Lima Norte. 
En los resultados, el indicador Aprender a investigar y realizar trabajos académicos $(\mathrm{B}=$ 0.345) es quien presentó mayor coeficiente y por ende contribuyó más al desarrollo académico en estudiantes de la universidad César Vallejo- Lima Norte. Así mismo, este indicador presenta un odds ratio $(\operatorname{Exp}(B))=1.412$, significando que es un indicador de riesgo que debe ser trabajada con mayor énfasis. De la misma forma, el indicador tomar conciencia de mi capacidad de aprendizaje para mejorar mi rendimiento $(\mathrm{B}=-0.281)$ es quien también presentó un mayor coeficiente, pero en segundo orden, así mismo aportó al desarrollo académico en estudiantes además es un indicador de protección, donde es necesario seguir reforzando este indicador para que siga mejorando.

\section{Discusión}

En la investigación se identificó que el $67 \%$ de los estudiantes consideraron la acción tutorial como adecuada, resultó en contraste con lo afirmado por al Álvarez (2013) al reflejar que la tutoría no se ha logrado implantar aún de forma plena, situación que alarmó a la condición universitaria en aquel contexto, considerando entonces a la acción tutorial como un elemento de suma influencia en su intervención. Marcelo (2015) afirmó que el acompañamiento tutorial realmente es importante durante el proceso de adaptación de los aprendices a la vida universitaria al responder a necesidades y requerimientos académicos, socioemocionales, culturales y de desarrollo personal, se confirmó de forma complementaria con el presente estudio que la acción si es adecuada para estudiantes universitarios en pleno proceso de formación y más aún como un intervención en beneficio de los estudiantes universitarios. También al entender, según los resultados descriptivos, que el 33\% del total de estudiantes de la universidad César Vallejo la acción tutorial es irregular e inadecuada, de forma complementaria Álvarez en su estudio publicado en el 2013, concluyó diciendo que ante la falta de herramientas adaptadas a la enseñanza superior fue una de las exigencias que se debió mejorar para atender demandas que hace el profesorado para planificar y poner en marcha planes de tutorías, es aquí donde se asumió como una de las razones del por qué la acción tutorial pueda tener un porcentaje de inadecuada o regular, y adoptarla como una medida a la que se deberá atender para mejores futuros resultados.

En el caso de la dimensión académica, los resultados descriptivos el $60 \%$ de los estudiantes consideraron al desarrollo académico en la acción tutorial como adecuado, mientras que el 11\% lo contrario, de manera similar Amor (2012) concluyó que el objetivo percibido de mayor utilidad en la acción tutorial es recibir orientación y asesoramiento en relación a su proceso de enseñanza y aprendizaje; ambos resultados reflejaron una cercanía de aceptación en el desarrollo académico, al respecto Gil, Martínez, Tunnicliffe y Moneo, (2013, p.69) afirmaron que el desarrollo académico, es la orientación al desarrollo de capacidades de aprendizaje autónomo, así como el desarrollo de la meta cognición para. En los mismos resultados se entendió en el estudio que el $40 \%$ consideró que consideró que el desarrollo académico de la acción tutorial que recibieron es regular e inadecuado, porcentaje que reflejó alguna deficiencia para una adecuada consideración en el desarrollo académico de la acción tutorial, en esto de forma complementaria Gil, Martínez, Tunnicliffe y Moneo (2013), concluyeron que los estudiantes meditaron en la importancia de atender a necesidades de desarrollo profesional a diferencia que las de desarrollo académico y personal; resultados que pudieron emitir alguna respuesta a resultados preliminares, que ya aquel porcentajes de inadecuado o regular se puede originar debido a que aquella muestra de estudiantes, consideran otras dimensiones de mayor necesidad, como en el caso de Gil, Martínez, Tunnicliffe y Moneo (2013) al considerar que la dimensión profesional tiene mayor consideración por sus estudiantes universitarios en España.

Los resultados también mostraron que el valor de significación observada (Sig.) es mayor al valor de significación teórica $\alpha=.05$ en todas las dimensiones aceptándose la hipótesis nula, por consiguiente no existieron diferencias en las dimensiones de la acción tutorial en estudiantes de la universidad César Vallejo- Lima Norte, motivo por el cual se rechazó la hipótesis general de investigación, ya que la dimensión predominante fue el desarrollo académico, pero al no ser significativa estadísticamente fue considerado un factor de riesgo ya que su valor de odds ratio < 
$\operatorname{Exp}(B)>$ es mayor a 1, convirtiéndose así en la dimensión que necesita mayor atención, ya que si no se vela por ella, la acción tutorial tendría mayores dificultades que los previstos; de manera complementaria y similar Amor (2016) concluyó que los estudiantes percibieron el desarrollo de la tutoría, como una actividad imprescindible en su proceder académico, confirmaron así que la acción tutorial es una actividad de relevancia para muchos estudiantes, y una de las mayores preocupaciones de los discentes al cual propusieron un mayor énfasis para la búsqueda de alternativas que den cobertura, y en este caso la acción tutorial es considerada una de ellas. Con la tesis de Amor (2012) se complementó el resultado inferencial relacionado al desarrollo personal, pues el indicador Poder conocerme a mí mismo $(B=0.296)$ presentó mayor coeficiente y por ende aportó más al desarrollo personal en los estudiantes de la muestra representativa, de esa forma se rechazó la hipótesis nula; por tal existieron diferencias en los indicadores de la dimensión desarrollo personal en estudiantes de la universidad César Vallejo- Lima Norte; y en el caso de Amor, concluyó que los estudiantes aceptaron que lo que debería cumplirse a través de las tutorías son el facilitar en el alumnado el desarrollo personal, favoreciendo su autoestima, motivación y autogestión, detectado y previniendo situaciones de riesgo (abandono/ fracaso académico), inclinándose en la búsqueda del bienestar estudiantil a partir de un bienestar personal.

En los mismos resultados de orden inferencial en relación a indicadores de la dimensión académica, reflejaron que se rechazaron la hipótesis nula. Por consiguiente existen diferencias en los indicadores de la dimensión desarrollo académico en estudiantes de la universidad César Vallejo- Lima Norte; siendo el indicador Aprender a investigar y realizar trabajos académicos (B $=0.345)$ es quien presenta mayor coeficiente y por ende aporta más al desarrollo académico en estudiantes de la universidad César Vallejo- Lima Norte, de forma complementaria Lemus, Torres, Serrano y Gúzman (2015) concluyó que el incorporar acciones de asesoría y tutoría entre pares pudo facilitar la comprensión del proceso por parte del estudiante mediante un plan de acción tutorial, estás afirmaciones conllevaron a nuevos horizontes pues si estudiantes universitarios están mencionando que dentro del desarrollo académico el aprender a investigar y realizar trabajos académicos puede estar en relación con tutorías entre pares (donde un estudiante de ciclos avanzados asesoran o ayudan el proceder académicos en estudiantes de primeros ciclos), acciones que en la universidad interviniente no trabaja este tipo de tutorías, pero el estudio lo planteó como una opción para nuevos resultados que estarían al orden de nuevas investigaciones y dar mayor profundidad a la misma en el desarrollo académico dentro de la acción tutorial. Dentro de los resultados que correspondieron a la dimensión de desarrollo profesional se rechazó la hipótesis nula. Por consiguiente, existieron diferencias en los indicadores de la dimensión desarrollo profesional en estudiantes de la universidad César Vallejo- Lima Norte. En este sentido el indicador Tomar conciencia de mi potencial para desarrollarme profesionalmente $(B=-0.512)$ presenta mayor coeficiente y por ende aporta más al desarrollo profesional en estudiantes, situación similar a los resultados y conclusiones de la tesis de Amor publicado en el 2012, al referir que los estudiantes aceptan que lo que debería cumplirse a través de las tutorías es desarrollar habilidades y destrezas en el desarrollo de su profesión; situación que reconoce a la acción tutorial como una labor amplia en muchas vertientes de trabajo, siendo el desarrollo profesional un campo de acción que todo discente desea optimalizar.

\section{Referencias}

Amor, M. (2012). La orientación y la tutoría universitaria como elementos para la calidad y la innovación en la educación superior. Modelo de Acción tutorial. (Tesis doctoral) Universidad de Córdoba, España. Recuperado de: http://helvia.uco.es/xmlui/bitstream/handle/10396/7665/556.pdf?sequence=1

Amor, M. (2016). Evaluación de la orientación y la tutoría en la facultad de ciencias de la educación de la universidad de Córdoba. Educatio Siglo XXI, 34(1), 93-112. Doi: https://doi.org/10.6018/j/253231

Álvarez, P. (2012). Los planes de tutoría de carrera: una estrategia para la orientación al estudiante en el marco del EEES. Educar, 48(2), 247-266. Recuperado de: https://www.raco.cat/index.php/Educar/article/view/259362 
Álvarez, P. (2013). La Función Tutorial del Profesorado Universitario: Una Nueva Competencia de la Labor Docente en el Contexto del EEES. Revista Portuguesa de Pedagogía, 47(2), pp. 85-106. Recuperado de: https://impactumjournals.uc.pt/rppedagogia/article/view/1907

Caldera, J., Carranza, M., Jiménez, A., \& Pérez, I. (2015). Actitudes de los estudiantes universitarios ante la tutoría. Diseño de una escala de medición. Revista de la Educación Superior, 44(1), pp. 103-124. Doi: https://doi.org/10.1016/j.resu.2015.04.004

Castellares, K. (2016). El docente universitario como tutor: La experiencia en los estudios generales. Revista PUCP En Blanco \& Negro, 7(2), 86-93. Recuperado de: http://revistas.pucp.edu.pe/index.php/enblancoynegro/article/view/16110

Gil, A., Martínez, A., Tunnicliffe, A., \& Moneo, J. (2013). Estudiantes universitarios y calidad del Plan de acción tutorial. Valoraciones y mejoras. Revista de Docencia Universitaria, 11(2), 63-87. Doi: https://doi.org/10.4995/redu.2013.5567

Lemus, R., Torres, F., Serrano, M., \& Gúzman G. (2015). La tutoría: eje fundamental para mejorar el rendimiento académico de alumnos de nivel superior. Innovación Educativa, 15(67), 90 - 110. Recuperado de: http://www.scielo.org.mx/pdf/ie/v15n67/v15n67a6.pdf

Lobato, C., \& Guerra, N. (2016). La tutoría en la educación superior en Iberoamérica: Avances y desafío. Educar, 52(2), 379-398. Recuperado de: https://ddd.uab.cat/pub/educar/educar_a2016v52n2/educar_a2016v52n2p379.pdf

Marcelo, N. (2015). Evaluación de la percepción de un programa tutorial dirigido a estudiantes de provincia en una universidad de lima. RIDU, 9(1), 1-16. Doi: https://doi.org/10.19083/ridu.9.401

Martínez, P., Pérez, J., \& Martínez, M. (2014). Una (re)visión de la tutoría universitaria: la percepción de estudiantes y tutores de estudios de Grado. REDU-Revista de Docencia Universitaria, 12(1), 269-305. Doi: https://doi.org/10.4995/redu.2014.6425

PRONABEC - Ministerio de Educación. (2012). Plan del Sistema de Tutoría para Instituciones de Educación Superior. Recuperado de: http://www.pronabec.gob.pe/inicio/publicaciones/documentos/plan_tutoria.pdf

Quintanal, J., \& Miraflores, E. (2013). Un modelo de tutoría en la Universidad del siglo XXI. Madrid: Editorial CCS.

Sánchez, C., \& Reyes, C. (2015). Metodología y Diseños en la investigación científica. Lima: Editorial Visión Universitaria. 